\title{
A CASE OF PYOSALPINX IN A NEWBORN INFANT
}

\author{
BY
}

\author{
ALBERT E. CLAIREAUX, M.B., Ch.B., M.R.C.P.Ed.; and \\ JAMES W. FARQUHAR, M.B., Ch.B. \\ (From the Department of Child Life and Health, University of Edinburgh)
}

Infections of the uterine adnexa are extremely uncommon in the neonatal period. Raso (1942) has reported one case of salpingitis in the newborn infant. He was unable to find a similar case in the literature at that time. His patient had suffered from severe umbilical sepsis and died from peritonitis on the eleventh day after birth. At necropsy there was marked umbilical sepsis, peritonitis, and early bilateral bronchopneumonia in addition to hepato-splenomegaly, which the author attributed to syphilis. The left Fallopian tube was found to be enlarged to two or three times the normal size in its middle third. There was a fibrino-purulent exudate in this region. On histological examination the surface epithelium of the mucous membrane was found to be relatively intact, but the corium was the site of an acute inflammatory reaction. The regional lymphatics were also full of pus. The serous coat was involved in the general peritonitis. Streptococci were found in the affected area. The opposite tube and uterus were quite healthy. The author suggested that the sepsis had spread to the peritoneum from the umbilical area and thence to the left tube via the lymphatics. He contrasts this pathogenesis with that of gonococcal salpingitis derived from an endometritis, and considers it resembles more closely septic lymphangitis of the Fallopian tubes in adults. Dunham (1933), in a review of sepsis in the newborn, reported several cases of peritonitis but in no instance were the tubes or ovaries involved. Martz and Foote (1938) have reviewed the literature concerning pyosalpinx in women. The earliest age of onset quoted therein is fifteen years. Dodek (1933) reported one case of a ruptured ovarian cyst in a newborn infant and considers the differential diagnosis.

The following case is one of neonatal pyosalpinx. It differs from the case reported by Raso (1942) in its pathogenesis but a streptococcus was isolated in this case also. Not only is the condition rare at this period, but, as will be shown, it can be a difficult one to diagnose.

\section{Case Report}

Baby McI., born on July 21, 1948, was the second child of a mother aged 28 years. The first child had been born spontaneously and is alive and well. When carrying this second child the mother had suffered from infective hepatitis. Following an antepartum haemorrhage she went into labour twelve weeks before term, and was spontaneously delivered of a small ( $3 \mathrm{lb} .9 \mathrm{oz}$.) limp and oedematous infant.

There was evidence of inhalation of amniotic fluid and prophylactic penicillin was given for a few days. Oedema was progressive and the fluid intake was accordingly reduced. No meconium was passed during the first thirty-six hours of life, but,- after rectal examination late on the second day, frequent and bulky meconium stools followed, including, on the third day, what appeared to be a cast of the bowel.

This relieved some abdominal distension which had been present, and on the tenth day the abdomen appeared normal and the infant was free from oedema, with the resultant loss of eleven ounces in weight since birth (19.3 per cent. fall).

Until two weeks old the baby had shown evidence of distressed respiration when removed from an atmosphere of continuous oxygen, but following that time oxygen was only given during or after feeding. The feeds consisted of expressed breast milk with additional amino acids.

On the twenty-sixth day the baby, which had shown some improvement and had begun to gain weight, was observed to have a small bead of yellow pus within the vulva in a position which occasioned some doubt as to whether it were of urethral or vaginal origin. A swab was taken and produced a mixed growth of B. coli, B. proteus, and a haemolytic streptococcus not of group A. A clear specimen of urine was obtained by catheterization and showed no pus cells on microscopic examination and grew a few colonies only of B. coli and enterococci. No further pus appeared but a short, forty-eight-hour, course of sulphadiazine was given.

The baby's abdomen was again noted to be distended on the twenty-ninth day, and on palpation of the abdomen a large mass was felt in the right loin. The tumour was firm and fixed. It extended to $5.0 \mathrm{~cm}$. below the costal margin, and it had a rounded lower pole. Above, it merged into the liver and could not be defined, and a rough estimation of $6.0 \mathrm{~cm}$. in length by $4.5 \mathrm{~cm}$. in breadth was made. Posteriorly the right loin was bulging and on percussion anteriorly the note was 
tympanitic until it encroached upon liver dulness above.

Liver and spleen appeared normal in size. Some dilatation of veins was visible on the anterior abdominal wall.

Radiological examination showed, on a straight film, slight elevation of the right dome of the diaphragm and a large area of irregular calcification $4.0 \times 2.0 \mathrm{~cm}$. in the right side of the abdomen lying approximately between the twelfth rib and a point a little above the iliac crest (fig. 1). It appeared to be too far lateral for kidney and extended below the shadow of the liver. A line of

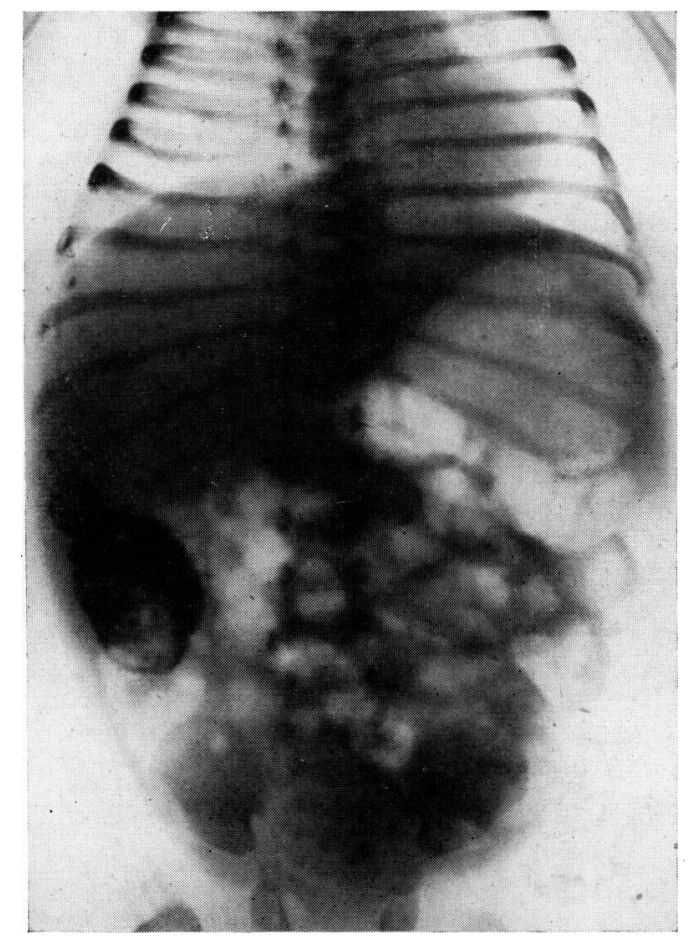

FIG. 1.-Radiograph of abdomen: antero-posterior view, showing calcified mass below right lobe of liver.

calcification appeared to extend upward almost to the level of the diaphragm. A small opacity was seen lying more medially at the level of the twelfth rib, and one or two doubtful shadows were seen below the left dome of the diaphragm. There was also a shadow in the pelvis to the left of the midline. A lateral radiograph of the abdomen showed the calcification to be lying in a somewhat intermediate position in the posterior half of the abdomen but some distance in front of the spine. The calcification in places appeared to consist of little seeds which did not alte- their position when the infant was inverted and were construed as tiny calculi fixed within a tumour mass or in the wall of a cyst. Excretion urography using intramuscular diodone failed to define the urinary tract at all clearly.

A tentative diagnosis of an embryoma in the region of the genital ridge was made and surgical opinion immediately sought.

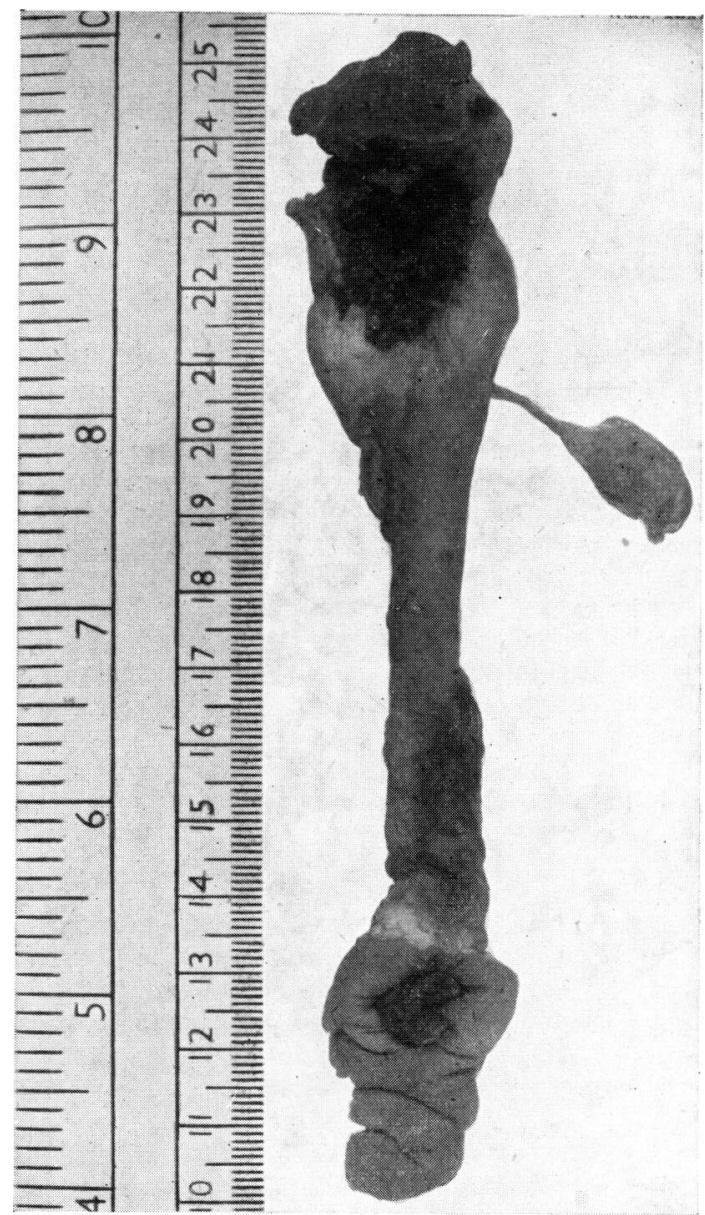

FIG. 2.- Specimen showing abscess cavity attached to liver. There is great elongation of uterus and vagina. The left tube and ovary are normal.

The following day distension was more marked and the baby's temperature was falling to subnormal level. The bowels were opening normally and the surgeon, after consultation with the radiologist, decided to observe rather than attempt immediate laparotomy. The baby's condition was poor and he could not agree with the provisional diagnosis, feeling it conceivable that the calcification might rather be taking place in a retroperitoneal haemorrhage oocasioned by trauma. 
During the next forty-eight hours, however, conditions deteriorated steadily. Distension of the abdomen was progressive and a ladder pattern of intestinal peristalsis was marked not only by its prominence on a poor abdominal wall, but by its great activity. Vomiting of bile commenced and became more frequent, and acidotic breathing appeared. Despite this the bowels continued to open two to five times daily and the stools were yellow or brownish yellow, containing no blood.

Surgical intervention was now quite ouc of the question but a last attempt was made to hydrate the baby and restore the electrolytic balance. Soluble sulphathiazole was given $(0 \cdot 125 \mathrm{~g}$. sixhourly).

On the night before death (the thirty-second day) very obvious gastric peristalsis accompanied the vomiting of bile, and the stools became more frequent and offensive. The following morning the baby was moribund, the respirations were laboured, the chest was full of coarse crepitations, and death ensued before mid-day.

POST-MORTEM REPORT: The body was that of a rather small premature female infant aged five weeks and weighing $3 \mathrm{lb} .4 \mathrm{oz}$. The atdomen was greatly distended and the skin was taut over the anterior abdominal wall. No congenital abnormalities were found on external examination.

The pleura was clear and glistening and there was no free fluid in the pleural sacs. The lungs were reddish pink in colour and quite well expanded. On section both lower lobes were moderately congested and a small quantity of whitish fluid could be expressed from the cut ends of the intra-pulmonary bronchi. As far as could be judged on macroscopic examination, there was no pneumonia. The pericardium was clear and glistening and there was no free fluid in the pericardial sac. The heart was of normal size and shape; the chambers had a normal appearance, and the erdocardium was healthy. Both the foramen ovale and ductus arteriosus were narrowly patent. No congenital abnormalities were present.

The peritoneum was rather dull and there was an extensive fibrinous peritonitis. This fibrinous exudate closely bourd the coils of intestine one to the other, but no actual pus was present in the peritoneal cavity. The stomach was rather distended and contained some partly digested food material ard mucus. The small bowel was rather dilated, possibly due to a paralytic ileus following on the peritonitis. The coils of intestine were firmly adherent to each other ard also to a mass of inflammatory tissue attached to the right lateral atdominal wall. The terminal ileum and the caecum and appendix were closely kourd down to the lateral atdominal wall in this region. The ascending colon, transverse colon, and pelvic colon were quite healthy. The rectum showed no change. Theliverwas of normal size and shape. The capsule was smooth except at its right inferior border where the liver was firmly attached to a mass of tissue lying close to the right lateral abdominal wall. The lower border of the right lobe of the liver was so firmly adherent that it could not be separated from the underlying tissue. On section the liver showed slight fatty change and also a slight infiltration of white matter at its lower border. This was probably a direct extension from the mass of inflammatory tissue. The spleen was three times normal size. The capsule was smooth and on section the organ was rather soft in consistency. The suprarenal glands had a normal appearance and the pancreas appeared to be healthy. The kidneys were small and showed a marked degree of foetal lobulation. The capsule was smooth and stripped easily, and on section a very mild degree of hydronephrosis was present in each kidney. Both ureters were also enlarged to about twice their normal diameter. This was due to mechanical pressure only. The bladder was not affected. After removal of these abdominal organs it was possible to attempt to dissect the mass of tissue lying anterior to the right kidney and firmly attached to the lower border of the liver and the right lateral abdominal wall. In doing so the mass was ruptured and a considerable quantity of thick greenish pus escaped. Specimens of the pus were taken for bacteriological examination. The tissue was then freed from the anterior abdominal wall and it now became obvious that it was continuous with the external genitalia, being connected to the latter by a long hollow muscular tube some $15 \mathrm{~cm}$. in length and $\frac{1}{2} \mathrm{~cm}$. in diameter. The upper mass of inflammatory tissue, the muscular tube and the external genitalia were removed en bloc. It was found that a probe could be passed from the vagina along this hollow tube to reach the lower pole of the inflammatory mass, thus accounting for the escape of pus per vaginam on one occasion. The upper inflammatory mass measured $3.5 \mathrm{~cm}$. in length by $2.5 \mathrm{~cm}$. in diameter. It consisted of thin muscular tissue and, after removal of pus, its inner aspect was seen to be lined with innumerable greenish black, small, hard calculi. Further examination showed that the inflammatory mass occupied the position of the right tube and ovary. No ovarian tissue was recognizable; it had been totally destroyed by the inflammatory reaction. The left tube and ovary were perfectly normal.

MicrosCOPICAL REPORT. The right Fallopian tube was the site of a chronic inflammatory process with a coincident acute inflammatory reaction. The mucous membrane had been destroyed and replaced by fibrous tissue. Some necrotic pockets of pus were undergoing calcification and were surrounded by foreign-body giant cells. In the more superficial layer there was a marked infiltration of polymorphonuclear leucocytes.

Liver. The liver cells were healthy and their normal lobular architecture had been preserved. The portal tracts had a normal appearance and the bile ducts were healthy. In one area, however, the capsule was replaced by a mass of acute inflammatory tissue. Without this zone of acute inflammatory 
tissue there was a zone of chronic inflammatory tissue and the Fallopian tube was attached to the adjacent liver. From this inflammatory zone there had been a direct extension of the infection along the portal tracts into the immediately adjacent liver tissue, and many lobules and portal tracts had been replaced by acute inflammatory infiltration with formation of small abscesses. The predominant cell in these areas was the polymorphonuclear leucocyte. The deeper portions of the liver had entirely escaped.

Abdominal tymph node. The capsule was normal. The germinal follicles were well developed and had a normal appearance. The sinuses were packed with lymphocytes and there was considerable reticulo-endothelial proliferation. The picture was that of chronic lymphadenitis.

Pancreas, lungs, kidneys and spleen showed no pathological change on histological examination.

BACTERIOLOGICAL REPORT (pus from abdomen). In the direct films were seen numerous pus cells and several Gram-negative bacilli and Gram-positive cocci, shown by culture to be B. coli and enterococci.

SUMmary. This baby had pyosalpinx of the right Fallopian tube with adhesions to the liver, local abscess formation in the liver, and fibrinous peritonitis.

\section{Discussion}

The abscess cavity, uterus and vagina, and external genitalia were removed en bloc at necropsy (fig. 2). Further dissection of this specimen after formol-fixation made the pathogenesis of the condition more obvious. The interior of the vagina and uterus were quite healthy. There was no evidence of endometritis, cervicitis, or vaginitis. The left Fallopian tube and ovary were also quite normal. The right Fallopian tube, however, was greatly distorted. It was bent back upon itself near its uterine end and firmly attached to the posterior uterine wall by numerous adhesions. The tube was dilated and hypertrophied in this region. It contained some thin watery pus, but the mucous membrane appeared to be almost normal, Distally the tube $(0.75 \mathrm{~cm}$. from its uterine orifice) opened directly into the abscess cavity, with which there was free communication. Infection had obviously settled down in the distal half of the right tube. This had proceeded to a pyosalpinx, and the large abscess thus formed destroyed all recognizable tissue, including the terminal portion of the tube and the ovary (fig. 3). The infection must have been a low-grade one, and organization proceeded pari passu with inflammatory destruction. This caused the formation of dense adhesions firmly binding down the abscess wall to the lateral abdominal wall and the inferior border of the liver. It may well be that when the infection began the right ovary was not fully descended and thus facilitated the attachment of the tube to the liver and structures in the right uipper quadrant of the abdomen.

The great elongation of the uterus $(3.5 \mathrm{~cm}$.) and vagina $(4.5 \mathrm{~cm}$.) was purely mechanical in origin and caused by the growth of these organs between two fixed points, namely the liver and the vulva. There was no evidence of embryological maldevelopment or infection in either uterus or vagina.

The source of infection is not easily determined. In view of the nature of the pathological and morphological changes, it would seem probable that the infection had commenced in utero. It is interesting to note that the mother had suffered from infective hepatitis during the early months of pregnancy. The organism responsible for the lesion would seem to be a haemolytic streptococcus not belonging to Lancefield's group A. Other organisms

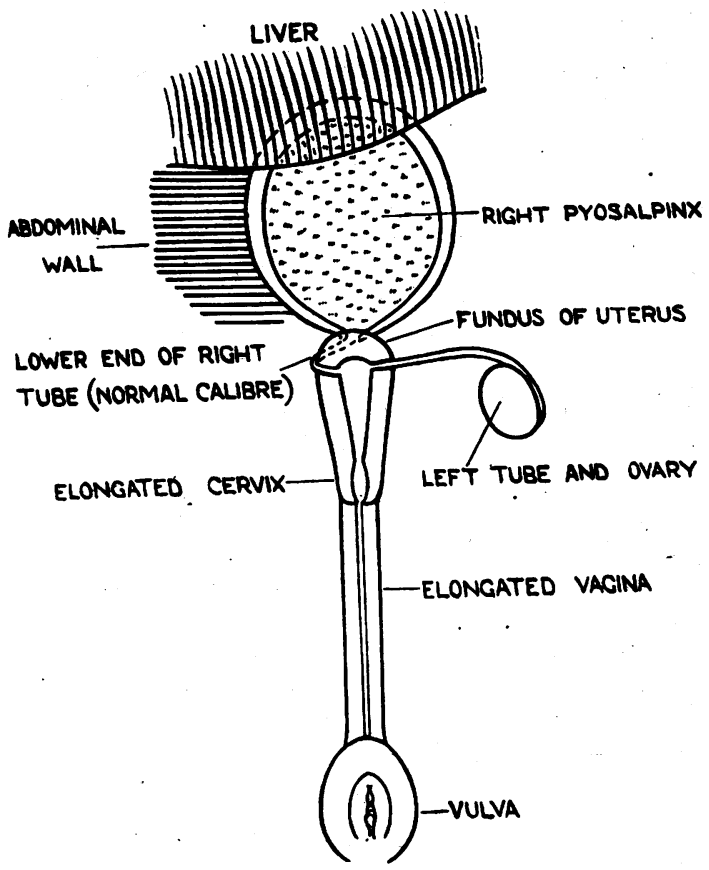

Fig. 3.-Diagrammatic representation of diseased tube and abscess cavity.

isolated from pus during life were probably contaminants from the napkin area. It would seem likely that the infection in this case was blood-borne.

Richmond and Moore (1947) have reported a case of intra-abdominal abscess causing intestinal obstruction in a newborn infant. They postulate the transplacental transmission of the organism concerned. It is interesting to note that in their case also the mother had suffered from jaundice during the fifth month of pregnancy.

The clinical signs presented are readily explicable once the pathology is known. It is regretted that such scant attention was paid to the single bead of pus found within the vulva on the twenty-sixth day. This, it was thought at the time, might be the product of a loculated pyonephrosis which had momentarily discharged some purulent material 
into the urinary tract. When the abdominal mass was no longer believed to be of renal origin, no serious attempt was made to explain this purulent discharge, which was regarded as a result of stasis.

Abdominal distension was due initially to the increasifing size of the abscess and its adjacent adhesions, but latterly was certainly due to a paralytic ileus with consequent intestinal obstruction, as the peristaltic waves were no longer transmitted onward.

Bowel motions continued since the large bowel was healthy and would consequently evacuate its contents. Veins are always clearly visible on the distended anterior abdominal wall of a small premature infant, but in this case the numerous adhesions involving the liver may have been responsible for an element of portal obstruction. The radiological appearances with apparent spread of calcification to liver and pelvis are now readily understood.

The case was an intriguing clinical and pathological problem and one to be remembered in the differential diagnosis of an intra-abdominal mass in the neonatal period.

We wish to express our thanks to Prof. R. W. B. Ellis for permission to publish this case.

\section{REFERENCES}

Dodek, S. M. (1933). Amer. J. Obstet. Gynec., 25, 914.

Dunham, E. C. (1933). Amer. J. Dis. Child., 45, 229.

Martz, H., and Foote, M. N. (1938). Amer. J. Obstet. Gynec., 36, 1009.

Raso, M. (1942). Arch. ostet. ginec., 6, 95.

Richmond, J. B., and Moore, H. R. (1947). J. Pediat., 31, 343. 